\title{
A Critical Review of Phenolic Compounds Extracted from the Bark of Woody Vascular Plants and Their Potential Biological Activity
}

\author{
Corneliu Tanase ${ }^{*}+\stackrel{\mathbb{D}}{ }$, Sanda Coșarcă ${ }^{\dagger}$ and Daniela-Lucia Muntean \\ Faculty of Pharmacy, University of Medicine, Pharmacy, Sciences and Technology of Târgu-Mures, \\ Gh. Marinescu Street No. 38, RO-540139 Tîrgu Mures,, Romania; sandacosarca31@gmail.com (S.C.); \\ danielaluciamuntean@yahoo.com (D.-L.M.) \\ * Correspondence: corneliu.tanase@umftgm.ro; Tel.: +40-079228277 \\ t These authors contributed equally to this work.
}

Academic Editors: Federica Pellati, Laura Mercolini and Roccaldo Sardella Received: 8 March 2019; Accepted: 24 March 2019; Published: 26 March 2019

\begin{abstract}
Polyphenols are one of the largest and most widespread groups of secondary metabolites in the plants world. These compounds are of particular interest due to their occurrence and the properties they possess. The main sources of phenolic compounds are fruits and vegetables, but lately, more and more studies refer to woody vascular plants, especially to bark, as an important source of phenolic compounds with a potential biological effect. This study aims to bring together information on the phenolic compounds present in the bark of woody vascular plants by discussing extraction methods, the chemical composition of the extracts and potential biological effects. The literature data used in this paper were collected via PubMed (2004-2019). Search terms were: bark, rhytidome, woody vascular plant, polyphenols, phenolic compounds, biologic activity, antioxidant, immunostimulatory, antimutagenic, antibacterial, anti-inflammatory, and antitumoral. This paper intends to highlight the fact that the polyphenolic extracts obtained from the bark of woody vascular plants represent sources of bioactive compounds with antioxidant, immunostimulatory, antimutagenic, antibacterial properties, etc. Future research directions should be directed towards identification and isolation of bioactive compounds. Consequently, biologically active compounds obtained from the bark of woody plants could be exploited on an industrial scale.
\end{abstract}

Keywords: bark; biological properties; extraction; polyphenols; rhytidome; woody vascular plants

\section{Introduction}

Current research is directed towards finding new sources of biologically active natural compounds with a wide range of applicability. Polyphenolic compounds are of particular interest due to their occurrence and properties. Phenolic compounds or polyphenols are one of the most frequent and widespread groups of substances in the world of plants, with more than 8000 identified phenolic structures [1]. These compounds can be found in almost all organs of a plant, and according to their structure, they have different functions ranging from skeletal constituents of different tissues to pigmentation of several plant organs [2]. Polyphenols are secondary metabolites essential for the growth and development of plants and their reproduction. Similarly, they help to control growth in diameter, pigmentation, and defence against various pathogens [3] or act as signalling molecules to distinguish symbionts [4]. These compounds, as natural antioxidants, have important properties that involve the inhibition of lipid peroxidation, inhibition of carcinogenesis, antimicrobial activity, direct constrictive action on capillaries, naturally occurring phytohormones, stabilisation of ascorbic acid, etc. [5]. 
The present paper is a critical review of the literature (2004-2019) on extraction methods of phenolic compounds from the bark of woody vascular plants, and their chemical composition, with an emphasis of their potential biological properties.

\section{The Bark of Woody Vascular Plants-Source of Phenolic Compounds}

The bark or rhytidome is a set of dead tissues, developed after the primary and secondary growth of bark (multiple layers of periderms), which together form the protective layers of branches and the trunks of woody vascular plants. The bark inhibits water loss through evaporation, has a protective role against overheating, frost, herbivores or infestation with parasites. The bark comprises up to $20 \%$ of the dry weight of woody vascular plants and contains polysaccharides, lignin, suberin, suberan, tannins or phenolic acids [6].

Currently, the woodworking industry produces a large amount of residue each year as a result of debarking woody vascular plants. Commonly, huge amounts of bark of woody plants can be found among wood wastes in the forest. These wastes are usually used for heating or as a cheap source of energy in cellulose factories, although these kinds of exploitations are not efficient and can lead to environmental problems [6].

The main sources of phenolic compounds are fruits and vegetables, but more studies refer to woody vascular plants, especially to bark, as an important source of phenolic compounds with a potential biological effect. Polyphenols, according to their chemical structure, are divided into sub-groups (Figure 1): phenolic acids (hydroxybenzoic and hydroxycinnamic acids), flavonoids (flavonols, flavones, flavanones, flavanonols, isoflavones, anthocyanidins, tannins), stilbenes (resveratrol) and lignans found in plants and foods of plant origin $[7,8]$.

Phenolic acids are one of the main classes of phenolic compounds found in plants and occur in the form of esters, glycosides or amides, but rarely in free form. The structural variation of phenolic acids depends on the number and position of hydroxyl groups on the aromatic ring. Phenolic acids have two distinctive structures: the hydroxycinnamic and hydroxybenzoic acid (Figure 1). The most common benzoic acids found in the bark of woody plants are vanillic, gallic, syringic and protocatechuic acid [9-11]. The most common cinnamic acids are $p$-coumaric, caffeic, ferulic and synaptic acid $[12,13]$.

Flavonoids are composed of two aromatic rings linked by a unit of three carbon atoms (C6-C3-C6). This carbon skeleton is the explanation for the chemical diversity of this family of compounds. The basic structures of flavonoids are aglycones but in plants, most of these are as glycosides [1]. The most common sub-groups of flavonoids found in bark of woody plants are flavonols (quercetin, kaempferol, myricetin, etc) [11,14-17], flavanonols (taxifolin), [14,16,18] flavones (apigenin, luteolin) flavanols [19] (catechin, epicatechin) $[10,14,16,20]$ and tannins $[18,19]$.

Stilbenes are phenolic compounds that contain two aromatic rings connected by a heterologous bridge. Resveratrol (3,5,4'-trihydroxystilbene) is the reference stilbene in grapes and wine [21] but it was identified in barks of Picea mariana (Mill.) Britton, Pinaceae [11] or Malus domestica Borkh, Rosaceae [22].

Lignans are dimers of phenylpropanoids, which result from the tail-to-tail binding of two coniferyl or sinapyl alcohol units. Examples of such compounds include isolariciresinol, secoisolariciresinol, lariciresinol, cedrusin and their glycosides [23], which present increasing interest in lignans especially due to their chemotherapeutic potential [24].

Bark contains large quantities of phenolic compounds and lignin. Thus, it can be considered as a possible renewable source of bioactive compounds, especially of aromatic substances. For example, Hofmann et al. [25] studied beech (Fagus sylvatica L., Fagaceae) bark and determined that the total polyphenol content was approximately $57 \mathrm{mg}$ gallic acid (GAE)/g dry bark units. The most efficient compounds with potential antioxidant activity in beech bark are epicatechin, coumaric acid, coniferin, quercetin, taxifolin-O-hexoside, coumaric acid-di-O-hexoside, syringic acid-di-O-hexoside, coniferyl alcohol-O-hexoside [26]. 
Another source of woody plant rich in phenolic compounds is the bark of black poplar (Populus nigra L., Salicaceae) with a total polyphenol content between 96.69-334.87 mg GAE/g dry bark units [27]. The bark of Schinopsis brasiliensis Engl., Anacardiaceae, is also an important source of polyphenols. The most important phenolic component identified as a chemical marker of $S$. brasiliensis is gallic acid [28].

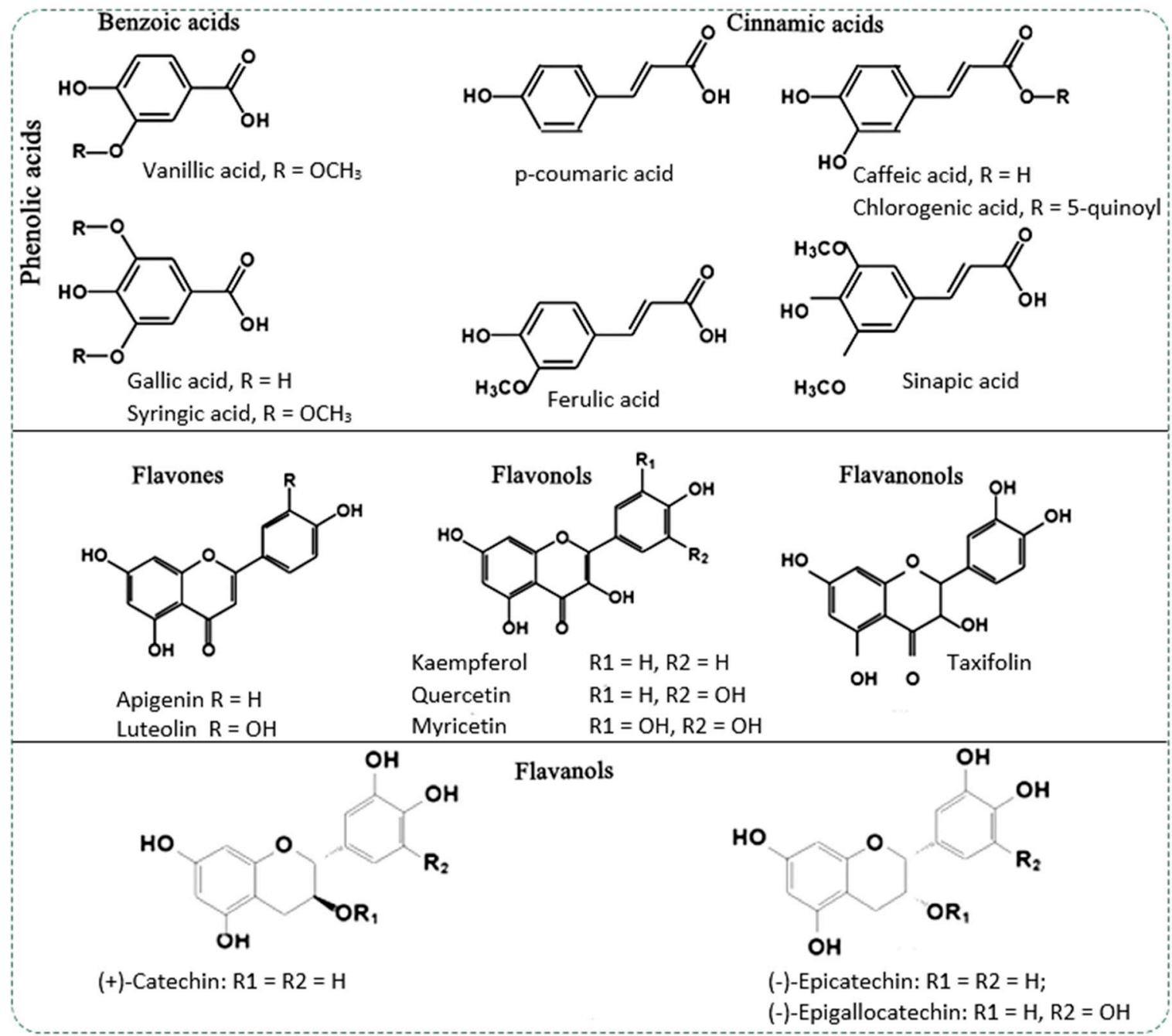

Figure 1. Typical phenolic compounds identified in the bark of woody vascular plants.

\section{Methods Used to Extract Phenolic Compounds from the Bark of Woody Vascular Plants}

The chemical composition of a plant product is determined by qualitative chemical analysis using various solvents for extraction. The choice of method and solvent used for extraction is a particularly important step to obtain an optimal concentration of natural compounds in the extract. It is important to select an efficient extraction method and proper work phases to assure high performance and increased stability of the extracted compounds [2].

The most commonly applied methods for the extraction of polyphenols use water in combination with organic solvents (acetone, ethanol, methanol, ethyl acetate) as per the type of polyphenols present in the bark of the plant [29]. Several authors reported increase of the extraction temperature could be correlated with increased efficiency [16]. Extraction time is a factor that should be taken into consideration as well. Prolonged extraction time can influence the oxidation process of polyphenols thus possibly decreasing the efficiency of the procedure and the type of extracted compounds [30].

Solid-liquid extraction is a common method used for obtaining polyphenols [31,32]; however, there may be various shortcomings such as long extraction time, increased quantity of solvent use, 
reduced potential to recover the solvent, which implies higher costs and higher toxicity. To improve extraction yield, time, and used solvent quantity, some unconventional (modern) methods such as ultrasonic extraction, microwave-assisted extraction, supercritical fluid extraction, pressurised liquid extraction or accelerated solvent extraction are preferred. The advantages of these methods compared to conventional methods (classical water bath extraction, Soxhlet extraction, and maceration) are the reduction of extraction time and quantity of required extraction solvent, as well as high reproducibility [33].

Ultrasound extraction is an effective alternative of conventional extraction methods, and the main advantage is its simplicity, the required equipment and the reduced extraction time [33,34]. However, in comparison with other modern methods, this one uses the highest amount of solvent and has the longest extraction time [33]. On the other hand, Chen et al. [35] demonstrated that ultrasonic extraction of the bark of Betula papyfera Marshall, Betulaceae, by using ethanol and water as a solvent has a maximum extraction yield at $180 \mathrm{~min}$ and $50{ }^{\circ} \mathrm{C}$. The optimal conditions for ultrasound extraction of polyphenols from the bark of Eucalyptus camaldulensis Dehnh., Myrtaceae, and Flourensia cernua DC., Asteraceae, are at $40-50{ }^{\circ} \mathrm{C}$ by using ethanol as extraction solvent [36].

During microwave extraction, the solid matter and solvent are subjected to microwave treatment, which accelerates the process of extraction due to the heating of the system. Thus, water within the vegetal matrix absorbs microwaves, and cell disruption occurs through internal superheating, which facilitates desorption of extractives from the vegetal matrix. This method uses polar solvent or a mixture of miscible polar solvents, because non-polar solvents do not or barely absorb microwave radiation. The advantages of this method lie in the fact that extraction time and the quantity of the solvent are reduced, whereas the efficiency of the extraction method is improved in comparison with conventional extraction methods [33]. Compared to other methods of polyphenol extraction, microwave-assisted extraction has proved to be efficient because of its shorter processing time [37]. It was observed that during the process of microwave extraction, time and microwave power are the main factors that influence efficiency significantly. It has also been noticed that the combination of miscible polar solvents improves the extraction yield [22,26,38,39].

Supercritical fluid extraction is an alternative solid-liquid extraction where the extraction solvent is replaced with a supercritical fluid (most commonly with carbon dioxide, but also with other materials such as nitric oxide, ethane, propane, $\mathrm{n}$-pentane, ammonia, and water). This is a relatively new method of processing solid and semi-solid substances, which has since become a specific technique referred to as supercritical fluid chromatography. The most important property of supercritical fluids during the extraction process is the ability to adjust solubility through physical parameters such as temperature and pressure, so that a supercritical fluid can extract a group of analytes of different polarities and molar masses in a more or less restricted fashion, and to reduce the volume of solvents used during extraction [40,41]. Supercritical fluid extraction was used to extract polyphenols from the bark of Hymenaea coubaril L., Fabaceae, by using $\mathrm{CO}_{2}, \mathrm{CO}_{2}+$ ethanol and $\mathrm{CO}_{2}+$ water as solvents, with the highest extraction yield being achieved with the combination of $\mathrm{CO}_{2}+$ water [42].

Accelerated solvent extraction is a new extraction method based on the use of high temperature and pressure to accelerate dissolution kinetics and to break the bonds of analyte-matrix interaction. Hence, this method is also referred to as pressurised fluid (solvent) extraction [30]. Moreover, by increasing the temperature the viscosity of the solvent decreases, which facilitates penetration of the solid matrix. This way, extraction time is reduced from tens of minutes to a couple of minutes, and extraction samples can be processed in small quantities. This method is an alternative of the Soxhlet or supercritical fluid extraction techniques [43].

Numerous studies (Table 1) have focussed on optimising methods of extracting polyphenols from the bark of woody vascular plants. In addition to conventional extraction methods, modern methods of polyphenol extraction are widely used as well $[27,39,44-46]$. Thus, for the same studied species, different values of total phenolic contents (TPC) can be obtained. For example, it was observed that for 
the Eucalyptus species the Soxhlet extraction method was preferred, thus obtaining a higher extraction yield of the total phenol content [47].

It has also been remarked that extraction temperature influences TPC and the type of extracted compounds. For example, Populus nigra L., Salicaceae, extracts obtained at temperatures above $200{ }^{\circ} \mathrm{C}$ displayed a higher content of flavonoid secondary metabolites and other polyphenols, and the level of antioxidant activity was higher than in the extracts obtained at temperatures below $180{ }^{\circ} \mathrm{C}$ [27].

Paz et al. [36] started researching four woody vascular plants Eucalyptus camaldulensis Dehnh., Myrtaceae, Flourensia cernua DC., Asteraceae, Jatropha dioica Sessé, Euphorbiaceae, and Turnera diffusa Willd. ex Schult., Passifloraceae. They used the ultrasound-assisted technique to obtain optimised extracts by adjusting the extraction time and solvent concentration (ethanol). Optimal conditions for extraction were created at $40 \mathrm{~min}$ of extraction time and $35 \%$ ethanol concentration.

Another intensively studied potential source of polyphenols is the bark of Picea abies L., Pinaceae. Researchers have attempted to optimize different methods of polyphenol extraction by changing the temperature, solid-liquid contact surface and extraction time in the presence of ultrasounds [48,49] and classical water bath extraction techniques [19,50]. For example, Lazar et al. [49] concluded that ultrasounds and temperature lead to significant effects on the polyphenolic compounds from spruce bark. Thus, the total content of polyphenols increased from $37.3 \mathrm{mg} \mathrm{GAE} \mathrm{g}^{-1} /$ spruce bark $/ 45^{\circ} \mathrm{C}$ to $43.1 \mathrm{mg} \mathrm{GAE} \mathrm{g}^{-1} /$ spruce bark / $60{ }^{\circ} \mathrm{C}$ [49].

In a study on the bark of Ulmus pumila L., Ulmaceae, conducted by Zhou et al. [51], the highest extraction yield was observed in the case of enzyme-assisted extraction (enzyme mixtures including cellulase, pectinase, and $\beta$-glucosidase) at $\mathrm{pH}=4.63,52.6^{\circ} \mathrm{C}$ and $62 \mathrm{~min}$ when the total polyphenol content was $16.04 \mathrm{mg}$ gallic acid/g dry matter.

Recent studies have highlighted the bark extracts of Terminalia arjuna (Roxb.) Wight \& Arn., Combretaceae, obtained with the use of various organic solvents, the highest polyphenol content extracted with butanol. The use of chloroform proved to have the lowest extraction capacity [52].

Table 1. Factors involved in different extraction methods according to the studied species.

\begin{tabular}{|c|c|c|c|c|c|}
\hline $\begin{array}{l}\text { Source of Bark: Scientific Name } \\
\text { (Family)-Commun Name }\end{array}$ & Extraction & Solvent & $\begin{array}{l}\text { Time } \\
(\mathrm{min})\end{array}$ & $\begin{array}{l}\text { Temperature } \\
{ }^{\circ} \mathrm{C}\end{array}$ & Reference \\
\hline Abies alba Mill (Pinaceae)-silver fir & CWBE & ethyl acetate & 120 & 70 & [53] \\
\hline Acacia confuse Merr. (Fabaceae) & CWBE & $\mathrm{NaOH} 1 \%$ & 60 & 100 & [54] \\
\hline $\begin{array}{c}\text { Acacia cornigera (L.) Willd. (Fabaceae), } \\
\text { bullhorn acacia }\end{array}$ & CWBE & $\begin{array}{l}\text { petroleum ether, } \\
\text { chloroform, } \\
\text { methanol }\end{array}$ & 4320 & RT & [12] \\
\hline $\begin{array}{c}\text { Acacia ferruginea DC. (Fabaceae)-rusty } \\
\text { acacia }\end{array}$ & SE & methanol 70\% & - & - & [55] \\
\hline $\begin{array}{l}\text { Acacia mearnsii Wild. (Fabaceae) } \\
\text { (Acacia mollissima)-black wattle }\end{array}$ & MAE & $\begin{array}{l}\text { methanol:water } \\
\text { 80:20 }\end{array}$ & - & - & {$[31]$} \\
\hline $\begin{array}{c}\text { Acacia nilotica L. (Fabaceae)-gum arabic } \\
\text { tree }\end{array}$ & CWBE & $\begin{array}{l}\text { methanol:ethanol, } \\
\text { acetone:water }\end{array}$ & 480 & RT & [29] \\
\hline $\begin{array}{c}\text { Acanthopanax leucorrhizus (Oliv.) Harms } \\
\text { (Araliaceae) }\end{array}$ & ME & ethanol 90\% & 1440 & RT & [44] \\
\hline $\begin{array}{l}\text { Acer saccharum Marshal } \\
\text { (Sapindaceae)-sugar maple }\end{array}$ & ME & ethanol 95\% & - & - & [56] \\
\hline Allophylus africanus Beauv. (Sapindaceae) & $\mathrm{CE}$ & water & 30 & - & [45] \\
\hline $\begin{array}{l}\text { Anacardium occidentale } \mathrm{L} . \\
\text { (Anacardiaceae)-cashew tree }\end{array}$ & ME & water & 120 & RT & [57] \\
\hline $\begin{array}{c}\text { Anogeissus leiocarpa DC. } \\
\text { (Combretaceae)-African birch }\end{array}$ & SE & ethanol & 300 & - & [58] \\
\hline $\begin{array}{c}\text { Azadirachta indica A.Juss. } \\
\text { (Meliaceae)-nimtree or Indian lilac }\end{array}$ & CWBE & $\begin{array}{l}\text { methanol:ethanol: } \\
\text { acetone:water }\end{array}$ & 480 & RT & [29] \\
\hline $\begin{array}{l}\text { Betula alleghaniensis Britt. } \\
\text { (Betulaceae)-yellow birch or golden birch }\end{array}$ & UAE & ethanol 95\% & - & - & [56] \\
\hline
\end{tabular}


Table 1. Cont.

\begin{tabular}{|c|c|c|c|c|c|}
\hline $\begin{array}{l}\text { Source of Bark: Scientific Name } \\
\text { (Family)-Commun Name }\end{array}$ & Extraction & Solvent & $\begin{array}{l}\text { Time } \\
(\min )\end{array}$ & $\begin{array}{l}\text { Temperature } \\
{ }^{\circ} \mathrm{C}\end{array}$ & Reference \\
\hline $\begin{array}{l}\text { Betula papyrifera Marshall } \\
\text { (Betulaceae)-Paper birch }\end{array}$ & UAE & ethanol:water 80:20 & 180 & 50 & [35] \\
\hline $\begin{array}{l}\text { Byrsonima crassifolia (L.) Kunth } \\
\text { (Malpighiaceae)-golden spoon }\end{array}$ & ME & $\begin{array}{l}\text { petroleum ether: } \\
\text { chloroform: } \\
\text { methanol }\end{array}$ & 4320 & RT & [12] \\
\hline Caraipa densifolia Mart. (Calophyllaceae) & SE & hexane:methanol & 180 & - & [59] \\
\hline $\begin{array}{l}\text { Cassia auriculata (L.) Roxb. } \\
\text { (Fabaceae)-matura tea tree }\end{array}$ & UAE & water & 300 & - & {$[60]$} \\
\hline \multirow{2}{*}{$\begin{array}{c}\text { Castanea sativa Mill. (Fagaceae)-sweet } \\
\text { chestnut }\end{array}$} & UAE & methanol & 30 & RT & [18] \\
\hline & SE & $\begin{array}{l}n \text {-hexane, acetone, } \\
\text { ethanol, methanol }\end{array}$ & 900 & - & {$[32]$} \\
\hline Cayratia pedata Lam. (Vitaceae) & UAE & methanol & 10 & RT & {$[61]$} \\
\hline $\begin{array}{l}\text { Chloroxylon swietenia DC. (Rutaceae)-East } \\
\text { Indian satinwood or buruta }\end{array}$ & UAE & methanol & 10 & RT & {$[61]$} \\
\hline Cinnamon sp. (Lauraceae) & CWBE & ethyl acetate & 600 & 30 & [62] \\
\hline $\begin{array}{c}\text { Coutarea hexandra (Jacq.) K. Schum } \\
\text { (Rubiaceae) }\end{array}$ & $\mathrm{ME}$ & ethanol 95\% & 10,080 & - & [63] \\
\hline Diotacanthus albiflorus Benth. (Acanthaceae) & UAE & methanol & 10 & RT & {$[61]$} \\
\hline $\begin{array}{l}\text { Drypetes klainei Pierre ex Pax } \\
\text { (Putranjivaceae) }\end{array}$ & ME & water & 180 & RT & [15] \\
\hline $\begin{array}{c}\text { Erythrina suberosa Roxb. (Fabaceae)-Corky } \\
\text { coral tree }\end{array}$ & CWBE & methanol & 1080 & RT & {$[64]$} \\
\hline $\begin{array}{l}\text { Eucalyptus camaldulensis Dehnh. } \\
\text { (Myrtaceae) }\end{array}$ & UAE & ethanol & - & $40-50$ & [36] \\
\hline \multirow{2}{*}{$\begin{array}{c}\text { Eucalyptus globulus Labill } \\
\text { (Myrtaceae)-Tasmanian bluegum, } \\
\text { blue gum }\end{array}$} & SE & $\begin{array}{l}n \text {-hexane, acetone, } \\
\text { ethanol, methanol }\end{array}$ & 900 & - & [32] \\
\hline & CWBE & $\begin{array}{l}\text { ethanol:water 80:20 } \\
(v / v)\end{array}$ & 360 & 82.5 & {$[65]$} \\
\hline $\begin{array}{l}\text { Eucalyptus grandis W.Hill ex. Maiden } \\
\text { (Myrtaceae)-rose gum }\end{array}$ & SE & dichloromethane & 360 & - & [47] \\
\hline $\begin{array}{l}\text { Eucalyptus maidenii F. Muell } \\
\text { (Myrtaceae)-Maiden's Gum }\end{array}$ & SE & dichloromethane & 360 & - & [47] \\
\hline $\begin{array}{l}\text { Eucalyptus sideroxylon A.Cunn. } \\
\text { (Myrtaceae)-mugga, red ironbark }\end{array}$ & UAE & ethanol:water & 60 & 50 & [66] \\
\hline $\begin{array}{l}\text { Eucalyptus urograndis (Myrtaceae)-Hybrid } \\
\text { E.grandis } \times \text { E. urophylla }\end{array}$ & SE & dichloromethane & 360 & - & [47] \\
\hline $\begin{array}{c}\text { Euclea undulata Thunb. } \\
\text { (Ebenaceae)-small-leaved guarri, } \\
\text { common guarri }\end{array}$ & CWBE & acetone & 4320 & RT & [67] \\
\hline Eucommia ulmoides Oliv. (Eucommiaceae) & $\mathrm{MAE}+\mathrm{UAE}$ & water, ethanol & $10-60$ & $20-60$ & [46] \\
\hline $\begin{array}{l}\text { Eugenia jambolana Lam. } \\
\text { (Myrtaceae)-Jamun, black plum }\end{array}$ & CWBE & $\begin{array}{c}\text { methanol } 80 \%, \\
\text { ethanol } 80 \%, \\
\text { acetone:water 80:20 }\end{array}$ & 480 & RT & [29] \\
\hline \multirow{3}{*}{$\begin{array}{c}\text { Fagus sylvatica } \mathrm{L} . \text { (Fagaceae)-common } \\
\text { beech }\end{array}$} & CWBE & $\begin{array}{c}\text { water, } \\
\text { methanol:water } \\
\text { 80:20, } \\
\text { ethanol:water 80:20 }\end{array}$ & $\begin{array}{l}120,300 \\
1440\end{array}$ & $\mathrm{RT}$ & \multirow{3}{*}{ [25] } \\
\hline & MAE & $\begin{array}{c}\text { water, } \\
\text { methanol:water } \\
\text { and ethanol:water } \\
(80: 20)\end{array}$ & 10,20 & $\begin{array}{l}60,80,100 \\
120\end{array}$ & \\
\hline & UAE & $\begin{array}{c}\text { water, } \\
\text { methanol:water } \\
\text { 80:20, } \\
\text { ethanol:water 80:20 }\end{array}$ & $10,20,30$ & RT & \\
\hline Ficus talboti King. (Moraceae)-talbot fig & SE & methanol & - & - & {$[68]$} \\
\hline $\begin{array}{c}\text { Flourensia cernua DC. } \\
\text { (Asteraceae)-American tarwort } \\
\text { and tarbush }\end{array}$ & UAE & ethanol & - & $40-50$ & [36] \\
\hline
\end{tabular}


Table 1. Cont.

\begin{tabular}{|c|c|c|c|c|c|}
\hline $\begin{array}{l}\text { Source of Bark: Scientific Name } \\
\text { (Family)-Commun Name }\end{array}$ & Extraction & Solvent & $\begin{array}{l}\text { Time } \\
(\mathrm{min})\end{array}$ & $\begin{array}{l}\text { Temperature } \\
{ }^{\circ} \mathrm{C}\end{array}$ & Reference \\
\hline $\begin{array}{l}\text { Guazuma ulmifolia Lam. (Malvaceae)-West } \\
\text { Indian elm or bay cedar }\end{array}$ & CWBE & $\begin{array}{l}\text { petroleum ether, } \\
\text { chloroform, } \\
\text { methanol }\end{array}$ & 4320 & RT & [69] \\
\hline $\begin{array}{c}\text { Goniothalamus velutinus Airy Shaw } \\
\text { (Annonaceae) }\end{array}$ & SE & absolute methanol & 600 & - & [70] \\
\hline Hugonia mystax Cav. (Linaceae) & CWBE & $\begin{array}{l}\text { distilled water, } \\
\text { methanol ethanol }\end{array}$ & - & RT & [13] \\
\hline Hymenaea courbaril L. (Fabaceae) & SFE & $\begin{array}{c}\mathrm{CO}_{2} \text { and water }(9: 1, \\
v / v)\end{array}$ & - & 56.85 & [42] \\
\hline $\begin{array}{c}\text { Jatropha dioica Sesse } \\
\text { (Euphorbiaceae)-leatherstem }\end{array}$ & UAE & ethanol & - & $40-50$ & [36] \\
\hline Lafoensia pacari A. St.-Hil (Lythraceae) & ME & absolute ethanol & 10,080 & RT & [20] \\
\hline $\begin{array}{l}\text { Larix laricina K. Koch (Pinaceae)-tamarack } \\
\text { or American larch }\end{array}$ & CWBE & ethanol 80\% & - & - & {$[71]$} \\
\hline $\begin{array}{c}\text { Liriodendron tulipifera } \mathrm{L} \text {. } \\
\text { (Magnoliaceae)-tulip tree, American tulip } \\
\text { tree, tulipwood }\end{array}$ & CWBE & oxalic acid $0.1 \mathrm{M}$ & 60 & 170 & {$[72]$} \\
\hline $\begin{array}{c}\text { Malus domestica Miller (Rosaceae)-apple } \\
\text { tree }\end{array}$ & $\begin{array}{l}\text { CWBE } \\
\text { MAE }\end{array}$ & $\begin{array}{c}\text { ethanol:water, 1:4 } \\
\text { ethanol:water, 60:40 } \\
v / v\end{array}$ & $\begin{array}{c}120 \\
20\end{array}$ & $\begin{array}{c}55 \\
100\end{array}$ & [22] \\
\hline Morus alba L. (Moraceae)-white mulberry & CWBE & methanol:water & - & - & [73] \\
\hline \multirow{4}{*}{ Picea abies L. (Pinaceae)-european spruce } & UAE & $\begin{array}{l}\text { ethanol:water } 50 \%, \\
70 \%(v / v)\end{array}$ & $30-60$ & $40-60$ & [48] \\
\hline & CWBE & water & $10-120$ & $60-90$ & [19] \\
\hline & CWBE & distilled water & 120 & 90 & {$[50]$} \\
\hline & UAE & $\begin{array}{l}\text { ethanol:water } 70 \% \\
(v / v)\end{array}$ & $5-75$ & $45-60$ & [49] \\
\hline $\begin{array}{l}\text { Picea mariana (Mill.) Britton (Pinaceae)-the } \\
\text { black spruce }\end{array}$ & SE & water & 60 & - & [11] \\
\hline Pinus sylvestris L. (Pinaceae)-Scots pine & CWBE & acetone:water & $3 \times 5$ & 100 & [74] \\
\hline Pinus brutia Tenore (Pinaceae)-Turkish pine & CWBE & distilled water & 60 & 70 & {$[75]$} \\
\hline \multirow{3}{*}{$\begin{array}{l}\text { Pinus pinaster Aiton (Pinaceae)-the } \\
\text { maritime pine, cluster pine }\end{array}$} & MAE & ethanol:water 80:20 & 30 & - & [38] \\
\hline & CWBE & $\begin{array}{l}\text { distilled water, } \\
\text { ethanol, methanol }\end{array}$ & - & - & {$[76]$} \\
\hline & CWBE & $\begin{array}{l}\text { water: } \mathrm{NaOH}: \mathrm{Na}_{2} \mathrm{SO}_{3} \text { : } \\
\qquad \mathrm{NaHSO}_{3}\end{array}$ & 120 & $70-80$ & [77] \\
\hline \multirow{3}{*}{$\begin{array}{l}\text { Pinus radiata } \text { D.Don (Pinaceae)-Monterey } \\
\text { pine, insignis pine or radiata pine }\end{array}$} & CWBE & Water & 60 & 100 & [78] \\
\hline & CWBE & deionized water & 30 & $95-99$ & [79] \\
\hline & CWBE & $\begin{array}{l}\text { ethanol:water, 3:1 } \\
(v / v)\end{array}$ & 120 & 120 & [10] \\
\hline Populus nigra L. (Salicaceae)-black poplar & $\begin{array}{l}\text { UAE } \\
\text { ME }\end{array}$ & $\begin{array}{l}\text { ethanol:water 70:30 } \\
\text { ethanol:water 70:30 }\end{array}$ & $\begin{array}{l}60 \\
-\end{array}$ & $\begin{array}{c}- \\
\text { RT }\end{array}$ & {$[27]$} \\
\hline $\begin{array}{c}\text { Punica granatum } \mathrm{L} . \\
\text { (Lythraceae)-Pomegranate }\end{array}$ & CWBE & methanol & - & RT & [80] \\
\hline Prunus domestica L. (Rosaceae)-Plums & UAE & $\begin{array}{l}7 \text { ethanol and } \mathrm{HCl} \\
1 \%, 2,6 \text {-di-tety- } \\
\text { butyl-4-methylphenol } \\
\text { (BHT) }\end{array}$ & - & - & [81] \\
\hline $\begin{array}{l}\text { Quercus robur L. (Fagaceae)-common oak, } \\
\text { pedunculate oak }\end{array}$ & MAE & $\begin{array}{l}\text { hydroalcoholic } \\
\text { solution of } \\
\text { methanol and } \\
\text { ethanol }\end{array}$ & $5-120$ & 100 & [82] \\
\hline $\begin{array}{c}\text { Rhus verniciflua (Stokes) F.Barkley } \\
\text { (Anacardiaceae)-Chinese lacquer tree }\end{array}$ & SE & ethanol & - & - & {$[83]$} \\
\hline Salix alba L. (Salicaceae)-white willow & ME & ethanol:water 70:30 & 1440 & - & [84] \\
\hline $\begin{array}{c}\text { Saraca asoca (Roxb.) Willd (Fabaceae)-the } \\
\text { ashoka tree }\end{array}$ & CWBE & methanol & 1440 & RT & [85] \\
\hline
\end{tabular}


Table 1. Cont.

\begin{tabular}{|c|c|c|c|c|c|}
\hline $\begin{array}{l}\text { Source of Bark: Scientific Name } \\
\text { (Family)-Commun Name }\end{array}$ & Extraction & Solvent & $\begin{array}{l}\text { Time } \\
(\mathrm{min})\end{array}$ & $\begin{array}{l}\text { Temperature } \\
{ }^{\circ} \mathrm{C}\end{array}$ & Reference \\
\hline $\begin{array}{l}\text { Schinopsis brasiliensis Engl. } \\
\text { (Anacardiaceae)-baraúna }\end{array}$ & ME & ethanol $90 \%$ & 7200 & RT & [47] \\
\hline $\begin{array}{l}\text { Sclerocarya birrea (A. Rich.) Hochst. } \\
\text { (Anacardiaceae)-marula }\end{array}$ & ME & distilled water & 2880 & RT & [86] \\
\hline Shorea roxburghii D.Don (Dipterocarpaceae) & CWBE & acetone:methanol & - & - & [87] \\
\hline Strychnos minor Dennst. (Loganiaceae) & UAE & methanol & 10 & RT & \multirow[b]{2}{*}{ [61] } \\
\hline $\begin{array}{l}\text { Strychnos nux-vomica Dennst. } \\
\text { (Loganiaceae)-the strychnine tree, nux } \\
\text { vomica, poison nut }\end{array}$ & UAE & methanol & 10 & RT & \\
\hline Sweetia panamensis Yakovlev (Fabaceae) & CWBE & $\begin{array}{l}\text { petroleum ether: } \\
\text { chloroform:methanol }\end{array}$ & 4320 & RT & [12] \\
\hline Terminalia brownie Fresen (Combretaceae) & SE & absolute ethanol & 300 & - & {$[58]$} \\
\hline \multirow{4}{*}{$\begin{array}{l}\text { Terminalia arjuna Wight \& Arn } \\
\text { (Combretaceae)-arjun tree }\end{array}$} & SE & $\begin{array}{l}\text { petroleum } \\
\text { ether:ethanol }\end{array}$ & - & $60-80$ & {$[88]$} \\
\hline & MAE & distilled water & 5 & - & [89] \\
\hline & ME & ethanol & 7200 & - & [90] \\
\hline & CWBE & $\begin{array}{l}\text { methanol:ethanol: } \\
\text { acetone:water }\end{array}$ & 480 & RT & [29] \\
\hline $\begin{array}{l}\text { Terminalia laxiflora Engl. \& Diels } \\
\text { (Combretaceae) }\end{array}$ & SE & absolute ethanol & 300 & - & [58] \\
\hline Trichilia catigua A.Juss. (Meliaceae) & CWBE & distilled water & 20 & 100 & [91] \\
\hline $\begin{array}{l}\text { Turnera diffusa Willd } \\
\text { (Passifloraceae)-Damiana }\end{array}$ & UAE & ethanol & - & $40-50$ & [36] \\
\hline \multirow{3}{*}{$\begin{array}{l}\text { Ulmus pumila L. (Ulmaceae)-the } \\
\text { Siberian elm }\end{array}$} & EAE & $\begin{array}{l}\text { cellulose, pectinase, } \\
\beta \text {-glucosidase }\end{array}$ & $60-90$ & $40-60$ & \multirow{3}{*}{ [51] } \\
\hline & UAE & ethanol $50 \%$ & $10-90$ & 52 & \\
\hline & CWBE & ethanol $50 \%$ & $10-90$ & 52 & \\
\hline Vitex doniana L. (Lamiaceae)-Black plum & ME & distilled water & 2880 & RT & [92] \\
\hline \multirow{4}{*}{ Ziziphus jujuba Mill. (Rhamnaceae)-Jujube } & CWBE & $\begin{array}{l}\text { ethanol, methanol, } \\
\text { hexane, acetone }\end{array}$ & 160 & 70 & \multirow{4}{*}{ [39] } \\
\hline & UAE & methanol & $20-60$ & RT & \\
\hline & SE & methanol & $40-140$ & 68 & \\
\hline & MAE & methanol & 4 & - & \\
\hline
\end{tabular}

RT-room temperature, CWBE-classic water bath extraction, ME-extraction by maceration, SE-Soxhlet extraction, UAE-ultrasound-assisted extraction, MAE—-microwave-assisted extraction, SFE-supercritical fluid extraction, EAE-enzymatic assisted extraction.

Hofmann et al. [25] aimed to optimise extraction methods according to the duration of exposure to ultrasounds and microwaves, solvents concentration and temperature. Thus, it was observed that the largest amount of polyphenols was obtained when the microwave-assisted extraction (MAE) technique was applied for $20 \mathrm{~min}$ by using ethanol and water as solvents. The extract with the most prominent antioxidant activity was obtained by the conventional extraction technique using water as solvent [25].

Vásquez et al. [14] identified significant amounts of polyphenols in the bark of Eucalyptus globulus Labill., Myrtaceae and Castanea sativa Mill., Fagaceae. They performed extractions using different solvents in different amounts. Regarding the total polyphenol content, the best extraction yield was obtained by using the conventional methanol-water extraction method for both the bark of E. globulus (TPC $=20.1 \mathrm{~g} \mathrm{GAE} / 100 \mathrm{~g}$ extract) and the bark of $C$. sativa $($ TPC $=59.7 \mathrm{~g} \mathrm{GAE} / 100 \mathrm{~g}$ extract), the only difference being the solvent ratio. They also determined the antioxidant activity (AOA) of the extracts obtained with different solvents and noticed that in the case of the bark of $C$. sativa, the extract with the best AOA was extracted with a solution of $2.5 \%$ sodium bisulfite, and for the bark of E. globulus, the methanol: water extraction type $(50: 50, v / v)$ provided the best results. 
Woody plant extracts like Jatropha dioica Sessé, Fluorensia cernua DC., Turnera diffusa Willd. ex Schult. and Eucalyptus camaldulensis Dehnh. studied by Paz et al. [36], showed the highest value of total polyphenol content at 40 min extraction time and 35\% ethanol concentration.

In a study regarding the composition and extraction yield of phenolic compounds of the species Acer saccharum Marshall, Sapindaceae and Betula alleghaniensis Britt., Betulaceae, we could observe similarities in the case of both ultrasound and maceration extraction, with the total polyphenol content being similar in the two species [56]. The bark of B. alleghaniensis was also studied by Diouf et al. [93] when the same extraction methods were used to determine the TPC and to identify triterpenic compounds such as lupenone, lupeol, betulinic acid, betulone, betulin and acetyl methyl betulinate.

In 2015, Enkhtaivan et al. [61] performed a comprehensive study on the total content of polyphenols in the bark of the following species Cayratia pedata (Lam.) Gagnep, Vitaceae, Chloroxylon swietenia DC., Rutaceae, Diotacanthus albiflorus Benth., Acanthaceae, Strychnos minor Dennst., Loganiaceae and Strychnos nux-vomica Dop., Loganiaceae. The results showed that the bark of $D$. albiflorus had the highest content of polyphenols ( $29.73 \mathrm{mg}$ GAE/g dry plant material). The polyphenol content of D. albiflorus and Strychnos nux-vomica was higher in their bark than in their leaves.

The phytochemical analysis of the bark of Acacia ferruginea DC., Myrtaceae, extract showed the presence of alkaloids, flavonoids, triterpene, tannins and the total polyphenolic content was about $438 \mathrm{mg}$ GAE/g dry plant material [55].

\section{Biological Effects of Extracts Obtained from the Bark of Woody Vascular Plants}

Phenolic compounds are known for their role in regulating the immune system, their anti-inflammatory effect, chemoprevention, neuroprotection, cardioprotection and in the treatment of diseases such as diabetes, Parkinson's disease and cancer; in addition to this, they also have antibacterial $[58,94]$ and antivirals effects [61]. Furthermore, the potential biological effects of some polyphenolic extracts obtained from the bark of woody vascular plants are presented (Table 2).

\subsection{Antioxidant Effect}

Polyphenols are compounds with one or more hydroxyl groups attached to the benzene ring. This structural feature provides a stronger acidic character to phenol than does to other alcohol groups. This chemical reactivity is responsible for the antioxidant character of polyphenols. The ability of polyphenols to capture free radicals is largely dependent on the number of hydroxyl groups $[14,84,87,95]$. There is a strong correlation between total polyphenol contents and antioxidant activity [90]. The main components possibly responsible for the antioxidant character of the T. arjuna extract were identified to be gallic acid, apigenin, luteolin, quercetin, epicatechin, ellagic acid [52]. In 2012, Santos et al. [47] studied three species of Eucalyptus, Myrtaceae, namely E. grandis W.Hill ex. Maiden, E. maidenii F. Muell and E. x urograndis. These species proved to have higher antioxidant potential than E. globulus [32]. The bark of E. x urograndis was found to have the highest antioxidant activity (IC50 $\left.=8.24 \mu \mathrm{g} \mathrm{mL}^{-1}\right)$ and the best extraction yield $(15.18 \%)$ compared to the other species included in the study $(10.54 \%$ for E. grandis and $13.23 \%$ for $E$. maidenii). Thus, the potential antioxidant effect of some polyphenolic global extracts obtained from the bark of woody vascular plants are presented in Table 2.

\subsection{Anti-Inflammatory Effect}

It has been demonstrated that besides their antioxidant effect, polyphenols reduce lipid peroxidation and DNA damage [96-98]. They also trigger a mechanism that blocks the overproduction of the tumour necrosis factor (TNF- $\alpha$ ), thus exerting an anti-inflammatory effect [27].

An increase in nitric oxide (NO) synthesis was observed in the inflamed tissues, and quercetin appeared to reduce the synthesis of nitrogen monoxide by inhibition of NO synthase [99]. For example, the Allophylus africanus P. Beauv., Sapindaceae, extract presented anti-inflammatory effects, successfully inhibiting the enzyme involved in the mechanism of inflammation, namely 5-lipoxygenase, which would 
be explained by the high quantity of flavons in the extract [45]. Other polyphenolic extracts obtained from the bark of woody vascular plants, with potential anti-inflammatory effect are presented in Table 2 .

\subsection{Antibacterial Effect}

Besides the antioxidant and anti-inflammatory activity of certain phenolic extracts, antimicrobial effects have also been observed. Several studies have been conducted on antibacterial activity. It was established that the ethanolic extract obtained from Picea abies L., Pinaceae, has antibacterial activity against Gram-positive (methicillin-resistant Staphylococcus aureus) and Gram-negative bacteria (Pseudomonas aeruginosa and Klebsiella pneumoniae) [100]. The study of the bark extract of Fagus sylvatica L., Fagaceae, underlined the antimicrobial activity against methicillin-resistant Staphylococcus aureus [101].

The methanolic extracts of some African herbs Terminalia arjuna Wight \& Arn, Combretaceae, T. brownie Fresen., Combretaceae and Anogeissus leiocarpus DC., Combretaceae, have revealed antimicrobial effects. In these extracts the combinations of different phenolic compounds such as ellagic acid, gallic acid, and ellagitannins were identified, but when they were separated and tested the antimicrobial effect decreased in comparison with the raw extract [58]. Another study on the antibacterial activity of T. arjuna revealed that the extracts of the bark presented the highest antibacterial activity. This effect has been tested against bacteria such as Bacillus subtilis, Staphylococcus aureus, Escherichia coli, Klebsiella pneumoniae, Pseudomonas aeruginosa and Salmonella typhi [90]. It was observed that the butanolic extract of bark was more effective in bacterial inhibition than extracts using water, chloroform or ethyl acetate as solvent, this being also correlated with higher values of TPC in butanolic extract, with $294 \mathrm{mg} / \mathrm{g}$ GAE versus $270 \mathrm{mg} / \mathrm{g}$ GAE in chloroform extract, and $189.9 \mathrm{mg} / \mathrm{g}$ GAE in aqueous extract.

Other polyphenolic extracts obtained from the bark of woody vascular plants, with potential antibacterial effect are presented in Table 2. The results of these studies open new research directions aimed at reducing pharmacological resistance of microorganisms to antibiotics by using plant phenolics.

\subsection{Other Effects}

Arunachalam and Parimelazhagan [68] researched the effects of the bark of Ficus talboti King, Moraceae, extract in diabetic rats with induced pathology. Their results were promising because they noticed that blood levels of triglycerides and cholesterol were reduced, body weight decreased, and the antidiabetic action was comparable to glibenclamide. They also observed that the activity of endogenous enzymes with antioxidant effect and insulin sensitivity of $\beta$-pancreatic cells increased. The authors suggested that the antidiabetic effect is due to the presence of routine, quercetin and kaempferol. The alcoholic extract of F. racemosa L. was proven to have a higher antioxidant effect than the aqueous extract [68].

The bark of Picea mariana (Mill.) Britton, Pinaceae, was studied and authors identified numerous phenolic components with important therapeutic action [11]. Thus, the phenolic compounds of lignan, neolignan, phenolic acids, and flavonoid classes were identified with important anti-inflammatory and antiproliferative activity with high potential of capitalisation in the pharmaceutical industry.

Erythrina suberosa Roxb., Fabaceae, an ornamental plant in India, has been studied concerning the cytotoxicity in leukaemia cell lines. Thus, it was concluded that $4^{\prime}$-Methoxy licoflavanone (MLF) and Alpinumisoflavone (AIF) inhibit the proliferation of HL-60 cells and induce their apoptosis [64].

Enkhtaivan et al. [61] investigated the bark of certain medicinal plant species like Cayratia pedata Lam., C. swietenia, D. albiflorus, S. minor, and S. nux-vomica L. and found a high antioxidant potential correlated with antiviral activity against H1N1 virus and cytotoxicity in Madin-Darby Canine Kidney (MDCK) cell lines.

Rhus verniciflua (Stokes) F. Barkley, Anacardiaceae, is a plant that has neuroprotective and anti-neuroinflammatory potential, and at the same time it enhances cognitive functions by protecting neurons against oxidative stress [102]. The neuroprotective and anti-inflammatory effect was 
tested in vitro, and the improvement of cognitive functions was highlighted by in vivo studies. The compounds responsible for the above mentioned effects appear to be the flavonoids named fisetin and butein, since fisetin increases the intracellular levels of glutathione and inhibits the activity of cyclooxygenase-2 (COX-2) and type II nitric oxide synthase (iNOS), which makes it an excellent therapeutic candidate for diminishing the progression of Alzheimer's disease and other neurodegenerative diseases. Another recently published study has highlighted the neuroprotective, antidepressant, anti-inflammatory and antioxidant effect of the aqueous phenolic extract from the bark of Trichilla catigua A. Juss., Meliaceae. The extract contained predominantly quinic acid derivatives, flavan-3-ols and phenylpropanoid substituted flavan-3-ols, largely responsible for the neuroprotective effect of the plant [91].

The hydroalcoholic extract of the bark of Acacia ferruginea DC., Myrtaceae, presents important therapeutic potential, considering that it is rich in flavonoids, triterpenoids, saponins, tannins and alkaloids. Faujdar et al. [55] studied this hydroalcoholic extract and confirmed its anti-inflammatory and anti-hemorrhoidal activity, but it has not been determined exactly which components of the extract have these specific effects.

The bark extract of T. catigua has been used empirically in the Brazilian traditional medicine for its neurostimulation and antidepressant effects. Recent studies have validated the traditional use and have demonstrated that the aqueous extract has been considered to have anti-inflammatory, antidepressant and neuroprotective effects due to the flavan-3-ol content and its phenylpropanoid derivatives [91].

Recent studies on raw extracts of the bark of S. brasilienisis have revealed its anti-inflammatory and antialgic effects. These effects appear to be due to the inhibition of central and peripheral pain transmission. Due to their mechanism of action, they inhibit the TNF- $\alpha$ proinflammatory factor by reducing the spread of inflammatory processes so that they neutralise reactive oxygen species, which also interfere with the mechanism of pain transmission. These effects of the extract are mainly attributed to gallic acid which is also a chemical marker of the species [16].

Table 2. The biological action of the extracts obtained from the bark of woody vascular plants.

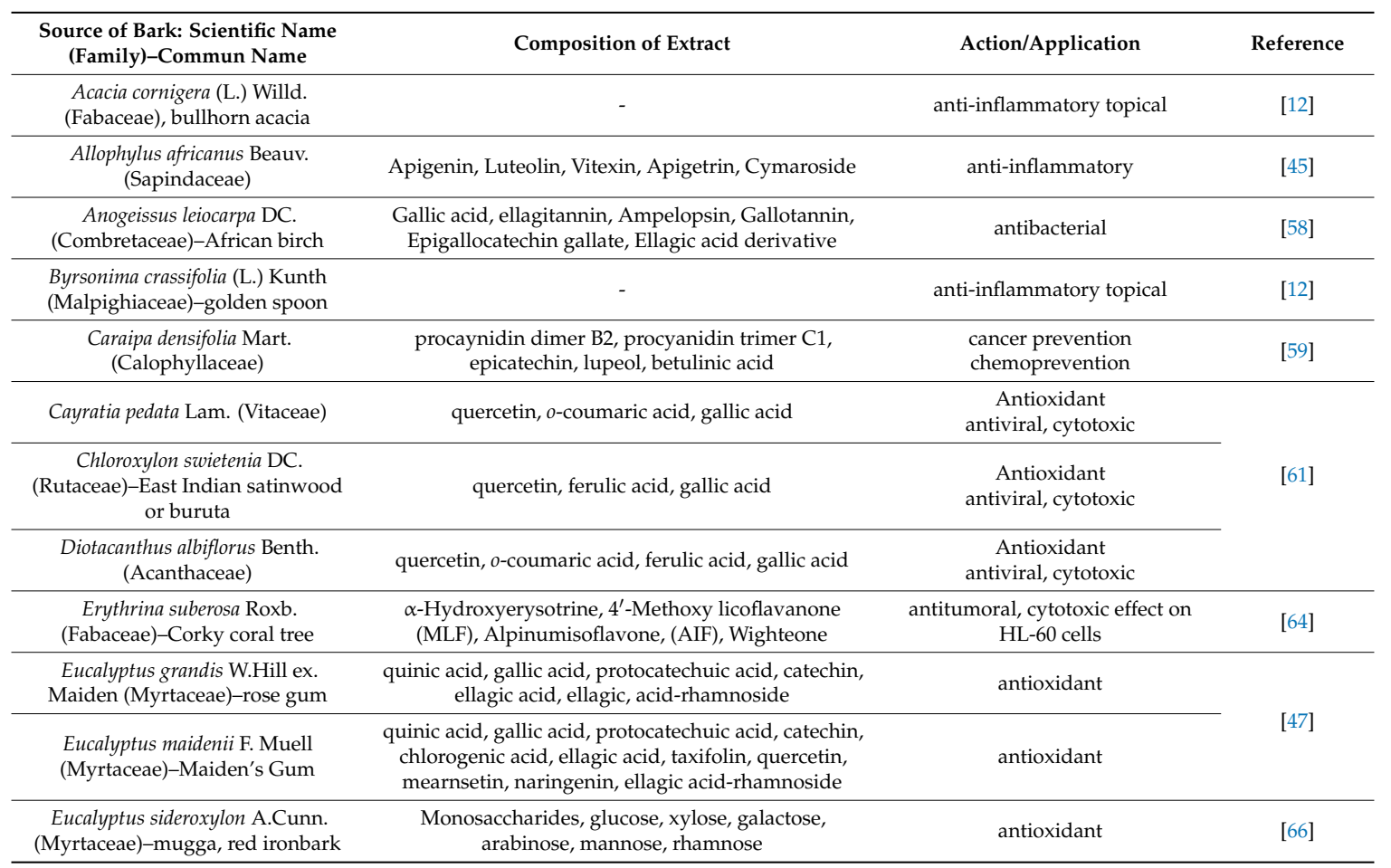


Table 2. Cont.

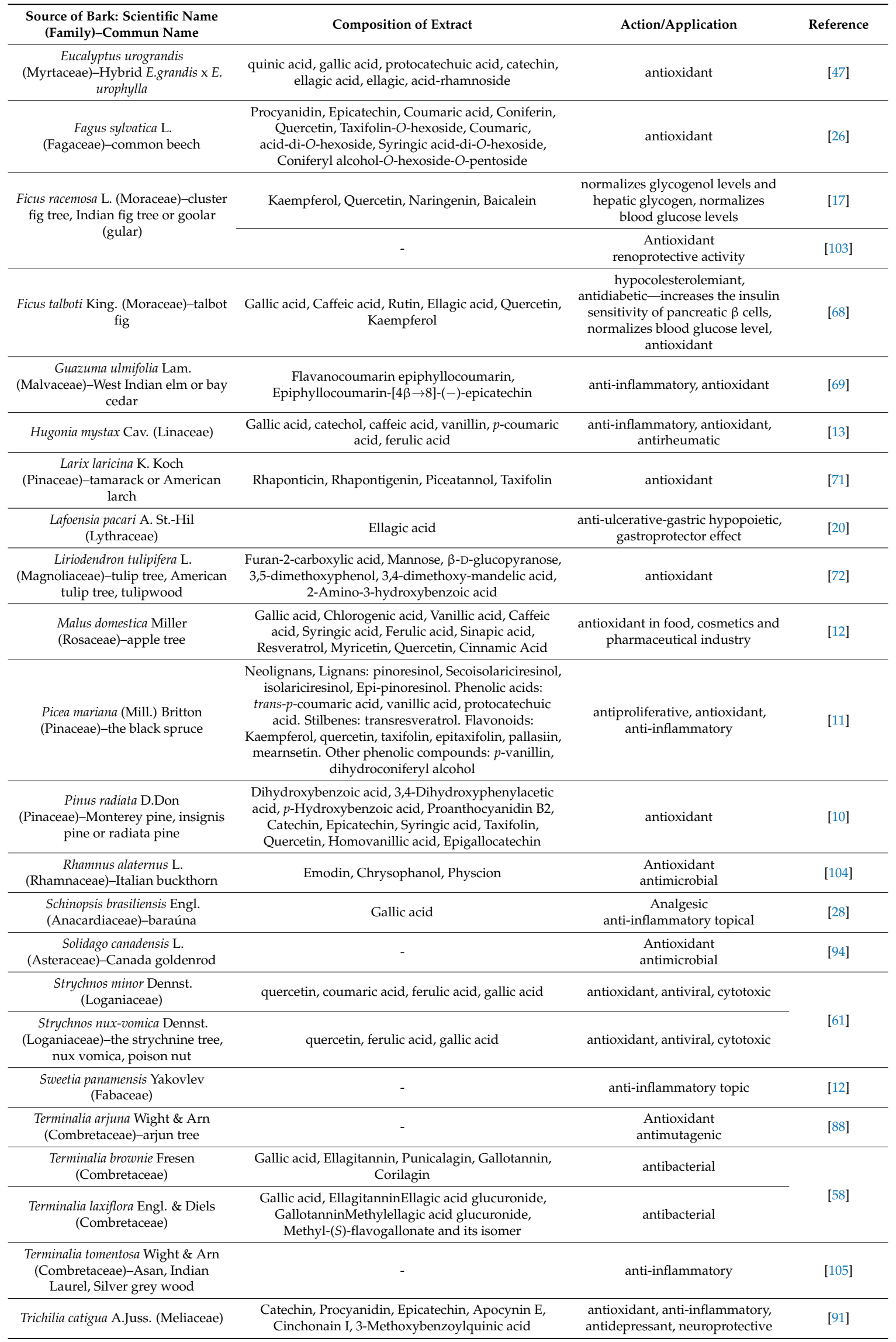




\section{Conclusions}

The bark of woody vascular plants is often considered a forest waste, but it can be an important source of bioactive compounds with a high potential for capitalisation. The large number of publications regarding the analysis of phenolic compounds extracted from the bark of woody vascular plants is testament to their importance and their value. Thus, many studies have focussed on optimising extraction methods and the identification of bioactive compounds. Numerous global extracts obtained from the bark of plants can have important biological effects such as antioxidant, antibacterial, anti-inflammatory, antitumoral, etc. Future research directions should be directed towards identification and isolation of bioactive compounds and the description of the mechanism of action of these compounds in living systems. Consequently, biologically active compounds obtained from the bark of woody plants could be exploited on an industrial scale.

Funding: This work was supported by a grant of Ministry of Research and Innovation, CNCS-UEFISCDI, project number PN-III-P1-1.1-PD-2016-0892, within PNCDI III.

Conflicts of Interest: The authors declare no conflict of interest.

\section{References}

1. Tsao, R. Chemistry and Biochemistry of Dietary Polyphenols. Nutrients 2010, 2, 1231-1246. [CrossRef]

2. Ignat, I.; Volf, I.; Popa, V.I. A critical review of methods for characterisation of polyphenolic compounds in fruits and vegetables. Food Chem. 2011, 126, 1821-1835. [CrossRef] [PubMed]

3. Popa, V.I. Wood bark as valuable raw material for compounds with biological activity. Celul. Şi Hârtie 2015, 64, 5-17.

4. Naczk, M.; Shahidi, F. Phenolics in cereals, fruits and vegetables: Occurrence, extraction and analysis. J. Pharm. Biomed. Anal. 2006, 41, 1523-1542. [CrossRef] [PubMed]

5. Tanase, C.; Boz, I.; Stingu, A.; Volf, I.; Popa, V.I. physiological and biochemical responses induced by spruce bark aqueous extract and deuterium depleted water with synergistic action in sunflower (Helianthus annuus 1.) plants. Ind. Crops Prod. 2014, 60, 160-167. [CrossRef]

6. Feng, S.; Cheng, S.; Yuan, Z.; Leitch, M.; Xu, C.C. Valorization of bark for chemicals and materials: A review. Renew. Sustain. Energy Rev. 2013, 26, 560-578. [CrossRef]

7. Dopico-García, M.; Fique, A.; Guerra, L.; Afonso, J.; Pereira, O.; Valentão, P.; Andrade, P.; Seabra, R. Principal components of phenolics to characterize red vinho verde grapes: Anthocyanins or non-coloured compounds? Talanta 2008, 75, 1190-1202. [CrossRef] [PubMed]

8. Oprică, L.; Socaciu, C. Metaboliţi Secundari din Plante: Origine, Structură, Funcţii; Editura Universităţii" Alexandru Ioan Cuza: Iasi, Romania, 2016.

9. Pereira, D.; Valentão, P.; Pereira, J.; Andrade, P. Phenolics: From Chemistry to Biology. Molecules 2009, 14, 2202-2211. [CrossRef]

10. Bocalandro, C.; Sanhueza, V.; Gómez-Caravaca, A.M.; González-Álvarez, J.; Fernández, K.; Roeckel, M.; Rodríguez-Estrada, M.T. Comparison of the composition of Pinus radiata bark extracts obtained at bench-and pilot-scales. Ind. Crops Prod. 2012, 38, 21-26. [CrossRef]

11. García-Pérez, M.-E.; Royer, M.; Herbette, G.; Desjardins, Y.; Pouliot, R.; Stevanovic, T. Picea mariana bark: A new source of trans-resveratrol and other bioactive polyphenols. Food Chem. 2012, 135, 1173-1182. [CrossRef]

12. Maldini, M.; Sosa, S.; Montoro, P.; Giangaspero, A.; Balick, M.J.; Pizza, C.; Loggia, R.D. Screening of the topical anti-inflammatory activity of the bark of Acacia cornigera Willdenow, Byrsonima crassifolia Kunth, Sweetia panamensis Yakovlev and the leaves of Sphagneticola trilobata Hitchcock. J. Ethnopharmacol. 2009, 122, 430-433. [CrossRef] [PubMed]

13. Pawar, S.S.; Dasgupta, D. Quantification of phenolic content from stem-bark and root of Hugonia mystax Linn. using RP-HPLC. J. King Saud Univ.-Sci. 2018, 30, 293-300. [CrossRef]

14. Vázquez, G.; Fontenla, E.; Santos, J.; Freire, M.S.; González-Álvarez, J.; Antorrena, G. Antioxidant activity and phenolic content of chestnut (Castanea sativa) shell and Eucalyptus (Eucalyptus globulus) bark extracts. Ind. Crops Prod. 2008, 28, 279-285. [CrossRef] 
15. Brusotti, G.; Andreola, F.; Sferrazza, G.; Grisoli, P.; Merelli, A.; della Cuna, F.R.; Calleri, E.; Nicotera, G.; Pierimarchi, P.; Serafino, A. In vitro evaluation of the wound healing activity of Drypetes klainei stem bark extracts. J. Ethnopharmacol. 2015, 175, 412-421. [CrossRef] [PubMed]

16. Chew, K.; Khoo, M.; Ng, S.; Thoo, Y.; Aida, W.W.; Ho, C. Effect of ethanol concentration, extraction time and extraction temperature on the recovery of phenolic compounds and antioxidant capacity of Orthosiphon stamineus extracts. Int. Food Res. J. 2011, 18, 1427.

17. Keshari, A.K.; Kumar, G.; Kushwaha, P.S.; Bhardwaj, M.; Kumar, P.; Rawat, A.; Kumar, D.; Prakash, A.; Ghosh, B.; Saha, S. Isolated flavonoids from Ficus racemosa stem bark possess antidiabetic, hypolipidemic and protective effects in albino wistar rats. J. Ethnopharmacol. 2016, 181, 252-262. [CrossRef] [PubMed]

18. Comandini, P.; Lerma-García, M.J.; Simó-Alfonso, E.F.; Toschi, T.G. Tannin analysis of chestnut bark samples (Castanea sativa Mill.) by HPLC-DAD-MS. Food Chem. 2014, 157, 290-295. [CrossRef]

19. Kemppainen, K.; Siika-aho, M.; Pattathil, S.; Giovando, S.; Kruus, K. Spruce bark as an industrial source of condensed tannins and non-cellulosic sugars. Ind. Crops Prod. 2014, 52, 158-168. [CrossRef]

20. Tamashiro Filho, P.; Olaitan, B.S.; de Almeida, D.A.T.; da Silva Lima, J.C.; Marson-Ascêncio, P.G.; Ascêncio, S.D.; Rios-Santos, F.; de Oliveira Martins, D.T. Evaluation of antiulcer activity and mechanism of action of methanol stem bark extract of Lafoensia pacari (Lytraceae) in experimental animals. J. Ethnopharmacol. 2012, 144, 497-505. [CrossRef] [PubMed]

21. Antoniolli, A.; Fontana, A.R.; Piccoli, P.; Bottini, R. Characterization of polyphenols and evaluation of antioxidant capacity in grape pomace of the Cv. Malbec. Food Chem. 2015, 178, 172-178. [CrossRef] [PubMed]

22. Moreira, M.M.; Barroso, M.F.; Boeykens, A.; Withouck, H.; Morais, S.; Delerue-Matos, C. Valorization of apple tree wood residues by polyphenols extraction: Comparison between conventional and microwave-assisted extraction. Ind. Crops Prod. 2017, 104, 210-220. [CrossRef]

23. Marinos, V.A.; Tate, M.E.; Williams, P.J. Lignan and phenylpropanoid glycerol glucosides in wine. Phytochemistry 1992, 31, 4307-4312. [CrossRef]

24. Saleem, M.; Kim, H.J.; Ali, M.S.; Lee, Y.S. An update on bioactive plant lignans. Nat. Prod. Rep. 2005, 22, 696-716. [CrossRef]

25. Hofmann, T.; Nebehaj, E.; Stefanovits-Bányai, É.; Albert, L. Antioxidant capacity and total phenol content of beech (Fagus sylvatica L.) bark extracts. Ind. Crops Prod. 2015, 77, 375-381. [CrossRef]

26. Hofmann, T.; Tálos-Nebehaj, E.; Albert, L.; Németh, L. Antioxidant efficiency of beech (Fagus sylvatica L.) bark polyphenols assessed by chemometric methods. Ind. Crops Prod. 2017, 108, 26-35. [CrossRef]

27. Todaro, L.; Russo, D.; Cetera, P.; Milella, L. Effects of thermo-vacuum treatment on secondary metabolite content and antioxidant activity of poplar (Populus nigra L.) wood extracts. Ind. Crops Prod. 2017, 109, 384-390. [CrossRef]

28. de Souza Santos, C.C.; Guilhon, C.C.; Moreno, D.S.A.; Alviano, C.S.; dos Santos Estevam, C.; Blank, A.F.; Fernandes, P.D. Anti-inflammatory, antinociceptive and antioxidant properties of Schinopsis brasiliensis bark. J. Ethnopharmacol. 2018, 213, 176-182. [CrossRef]

29. Sultana, B.; Anwar, F.; Przybylski, R. Antioxidant activity of phenolic components present in barks of Azadirachta indica, Terminalia arjuna, Acacia nilotica, and Eugenia jambolana trees. Food Chem. 2007, 104, 1106-1114. [CrossRef]

30. Dai, J.; Mumper, R.J. Plant phenolics: Extraction, analysis and their antioxidant and anticancer properties. Molecules 2010, 15, 7313-7352. [CrossRef] [PubMed]

31. Rhazi, N.; Hannache, H.; Oumam, M.; Sesbou, A.; Charrier, B.; Pizzi, A.; Charrier-El Bouhtoury, F. Green extraction process of tannins obtained from moroccan Acacia mollissima barks by microwave: Modeling and optimization of the process using the response surface methodology RSM. Arab. J. Chem.. in press. [CrossRef]

32. Koleva, V.; Simeonov, E. Solid liquid extraction of phenolic and flavonoid compounds from Cotinus coggygria and concentration by nanofiltration. Chem. Biochem. Eng. Q. 2014, 28, 545-551. [CrossRef]

33. Chemat, F.; Khan, M.K. Applications of ultrasound in food technology: Processing, preservation and extraction. Ultrason. Sonochem. 2011, 18, 813-835. [CrossRef] [PubMed]

34. Liu, E.-H.; Qi, L.-W.; Cao, J.; Li, P.; Li, C.-Y.; Peng, Y.-B. Advances of modern chromatographic and electrophoretic methods in separation and analysis of flavonoids. Molecules 2008, 13, 2521-2544. [CrossRef] [PubMed] 
35. Chen, Q.; Fu, M.; Liu, J.; Zhang, H.; He, G.; Ruan, H. Optimization of ultrasonic-assisted extraction (UAE) of betulin from white birch bark using response surface methodology. Ultrason. Sonochem. 2009, 16, 599-604. [CrossRef]

36. Paz, J.E.W.; Márquez, D.B.M.; Ávila, G.C.M.; Cerda, R.E.B.; Aguilar, C.N. Ultrasound-assisted extraction of polyphenols from native plants in the mexican desert. Ultrason. Sonochem. 2015, 22, 474-481.

37. Tatke, P.; Jaiswal, Y. An Overview of microwave assisted extraction and its applications in herbal drug research. Res. J. Med. Plant 2011, 5, 21-31. [CrossRef]

38. Chupin, L.; Maunu, S.; Reynaud, S.; Pizzi, A.; Charrier, B.; Bouhtoury, F.C.-E. Microwave assisted extraction of maritime pine (Pinus pinaster) bark: Impact of particle size and characterization. Ind. Crops Prod. 2015, 65, 142-149. [CrossRef]

39. Dubey, K.K.; Goel, N. Evaluation and optimization of downstream process parameters for extraction of betulinic acid from the bark of Ziziphus jujubae L. Sci. World J. 2013, 2013, 469674. [CrossRef]

40. Seabra, I.; Braga, M.; de Sousa, H. Statistical mixture design investigation of $\mathrm{CO}_{2}$-ethanol- $\mathrm{H}_{2} \mathrm{O}$ pressurized solvent extractions from tara seed coat. J. Supercrit. Fluids 2012, 64, 9-18. [CrossRef]

41. Zougagh, M.; Valcárcel, M.; Ríos, A. Supercritical fluid extraction: A critical review of its analytical usefulness. TrAC Trends Anal. Chem. 2004, 23, 399-405. [CrossRef]

42. Veggi, P.C.; Prado, J.M.; Bataglion, G.A.; Eberlin, M.N.; Meireles, M.A.A. Obtaining phenolic compounds from jatoba (Hymenaea courbaril L.) bark by supercritical fluid extraction. J. Supercrit. Fluids 2014, 89, 68-77. [CrossRef]

43. Mustafa, A.; Turner, C. Pressurized liquid extraction as a green approach in food and herbal plants extraction: A review. Anal. Chim. Acta 2011, 703, 8-18. [CrossRef]

44. Hu, H.-B.; Liang, H.-P.; Li, H.-M.; Yuan, R.-N.; Sun, J.; Zhang, L.-L.; Han, M.-H.; Wu, Y. Isolation, purification, characterization and antioxidant activity of polysaccharides from the stem barks of Acanthopanax leucorrhizus. Carbohydr. Polym. 2018, 196, 359-367. [CrossRef] [PubMed]

45. Ferreres, F.; Gomes, N.G.; Valentão, P.; Pereira, D.M.; Gil-Izquierdo, A.; Araújo, L.; Silva, T.C.; Andrade, P.B. Leaves and stem bark from Allophylus africanus Beauv.: An approach to anti-inflammatory properties and characterization of their flavonoid profile. Food Chem. Toxicol. 2018, 118, 430-438. [CrossRef] [PubMed]

46. Li, L.; Guo, Y.; Zhao, L.; Zu, Y.; Gu, H.; Yang, L. Enzymatic hydrolysis and simultaneous extraction for preparation of genipin from bark of Eucommia ulmoides after ultrasound, microwave pretreatment. Molecules 2015, 20, 18717-18731. [CrossRef] [PubMed]

47. Santos, S.A.; Villaverde, J.J.; Freire, C.S.; Domingues, M.R.M.; Neto, C.P.; Silvestre, A.J. Phenolic composition and antioxidant activity of Eucalyptus grandis, E. urograndis (E. grandis $\times$ E. urophylla) and E. maidenii bark extracts. Ind. Crops Prod. 2012, 39, 120-127. [CrossRef]

48. Ghitescu, R.-E.; Volf, I.; Carausu, C.; Bühlmann, A.-M.; Gilca, I.A.; Popa, V.I. Optimization of ultrasound-assisted extraction of polyphenols from spruce wood bark. Ultrason. Sonochem. 2015, 22, 535-541. [CrossRef] [PubMed]

49. Lazar, L.; Talmaciu, A.I.; Volf, I.; Popa, V.I. Kinetic Modeling of the ultrasound-assisted extraction of polyphenols from Picea abies bark. Ultrason. Sonochem. 2016, 32, 191-197. [CrossRef] [PubMed]

50. Lacoste, C.; Čop, M.; Kemppainen, K.; Giovando, S.; Pizzi, A.; Laborie, M.-P.; Sernek, M.; Celzard, A. Biobased foams from condensed tannin extracts from norway spruce (Picea abies) bark. Ind. Crops Prod. 2015, 73, 144-153. [CrossRef]

51. Zhou, Z.; Shao, H.; Han, X.; Wang, K.; Gong, C.; Yang, X. The extraction efficiency enhancement of polyphenols from Ulmus pumila L. barks by trienzyme-assisted extraction. Ind. Crops Prod. 2017, 97, 401-408. [CrossRef]

52. Ambika, S.; Chauhan, S. Activity-guided isolation of antioxidants from the leaves of Terminalia arjuna. Nat. Prod. Res. 2014, 28, 760-763.

53. Benković, E.T.; Grohar, T.; Žigon, D.; Švajger, U.; Janeš, D.; Kreft, S.; Štrukelj, B. Chemical composition of the silver fir (Abies alba) bark extract abigenol and its antioxidant activity. Ind. Crops Prod. 2014, 52, $23-28$. [CrossRef]

54. Lee, W.-J.; Lan, W.-C. Properties of resorcinol-tannin-formaldehyde copolymer resins prepared from the bark extracts of taiwan acacia and china fir. Bioresour. Technol. 2006, 97, 257-264. [CrossRef]

55. Faujdar, S.; Sati, B.; Sharma, S.; Pathak, A.; Paliwal, S.K. Phytochemical evaluation and anti-hemorrhoidal activity of bark of Acacia ferruginea DC. J. Tradit. Complement. Med. 2019, 9, 85-89. [CrossRef] 
56. St-Pierre, F.; Achim, A.; Stevanovic, T. Composition of ethanolic extracts of wood and bark from Acer saccharum and Betula alleghaniensis trees of different vigor classes. Ind. Crops Prod. 2013, 41, 179-187. [CrossRef]

57. Encarnação, S.; de Mello-Sampayo, C.; Graca, N.A.; Catarino, L.; da Silva, I.B.M.; Lima, B.S.; Silva, O.M.D. Total Phenolic content, antioxidant activity and pre-clinical safety evaluation of an Anacardium occidentale stem bark portuguese hypoglycemic traditional herbal preparation. Ind. Crops Prod. 2016, 82, 171-178. [CrossRef]

58. Salih, E.; Kanninen, M.; Sipi, M.; Luukkanen, O.; Hiltunen, R.; Vuorela, H.; Julkunen-Tiitto, R.; Fyhrquist, P. Tannins, flavonoids and stilbenes in extracts of african savanna woodland trees Terminalia brownii, Terminalia laxiflora and Anogeissus leiocarpus showing promising antibacterial potential. South Afr. J. Bot. 2017, 108, 370-386. [CrossRef]

59. Da Silveira, C.; Trevisan, M.; Rios, J.; Erben, G.; Haubner, R.; Pfundstein, B.; Owen, R. Secondary plant substances in various extracts of the leaves, fruits, stem and bark of Caraipa densifolia Mart. Food Chem. Toxicol. 2010, 48, 1597-1606. [CrossRef] [PubMed]

60. Sivakumar, V.; Ilanhtiraiyan, S.; Ilayaraja, K.; Ashly, A.; Hariharan, S. Influence of ultrasound on avaram bark (Cassia auriculata) tannin extraction and tanning. Chem. Eng. Res. Des. 2014, 92, 1827-1833. [CrossRef]

61. Enkhtaivan, G.; John, K.M.; Ayyanar, M.; Sekar, T.; Jin, K.-J.; Kim, D.H. Anti-Influenza (H1N1) potential of leaf and stem bark extracts of selected medicinal plants of south india. Saudi J. Biol. Sci. 2015, 22, 532-538. [CrossRef] [PubMed]

62. Kandhare, A.D.; Bodhankar, S.L.; Singh, V.; Mohan, V.; Thakurdesai, P.A. Anti-asthmatic effects of type-a procyanidine polyphenols from Cinnamon bark in ovalbumin-induced airway hyperresponsiveness in laboratory animals. Biomed. Aging Pathol. 2013, 3, 23-30. [CrossRef]

63. Nunes, L.G.; Gontijo, D.C.; Souza, C.J.; Fietto, L.G.; Carvalho, A.F.; Leite, J.P.V. The mutagenic, DNA-damaging and antioxidative properties of bark and leaf extracts from Coutarea hexandra (Jacq.) K. Schum. Environ. Toxicol. Pharmacol. 2012, 33, 297-303. [CrossRef] [PubMed]

64. Kumar, S.; Pathania, A.S.; Saxena, A.; Vishwakarma, R.; Ali, A.; Bhushan, S. The anticancer potential of flavonoids isolated from the stem bark of Erythrina suberosa through induction of apoptosis and inhibition of STAT signaling pathway in human leukemia HL-60 Cells. Chem. Biol. Interact. 2013, 205, 128-137. [CrossRef] [PubMed]

65. Baptista, E.A.; Pinto, P.C.; Mota, I.F.; Loureiro, J.M.; Rodrigues, A.E. Ultrafiltration of ethanol/water extract of Eucalyptus globulus bark: Resistance and cake build up analysis. Sep. Purif. Technol. 2015, 144, 256-266. [CrossRef]

66. Miranda, I.; Lima, L.; Quilhó, T.; Knapic, S.; Pereira, H. The bark of Eucalyptus sideroxylon as a source of phenolic extracts with anti-oxidant properties. Ind. Crops Prod. 2016, 82, 81-87. [CrossRef]

67. Deutschländer, M.; Lall, N.; Van de Venter, M.; Hussein, A.A. Hypoglycemic evaluation of a new triterpene and other compounds isolated from Euclea undulata Thunb. var. myrtina (Ebenaceae) root bark. J. Ethnopharmacol. 2011, 133, 1091-1095. [CrossRef]

68. Arunachalam, K.; Parimelazhagan, T. Antidiabetic and enzymatic antioxidant properties from methanol extract of Ficus talboti bark on diabetic rats induced by streptozotocin. Asian Pac. J. Reprod. 2014, 3, 97-105. [CrossRef]

69. Maldini, M.; Di Micco, S.; Montoro, P.; Darra, E.; Mariotto, S.; Bifulco, G.; Pizza, C.; Piacente, S. Flavanocoumarins from Guazuma ulmifolia bark and evaluation of their affinity for STAT1. Phytochemistry 2013, 86, 64-71. [CrossRef]

70. Iqbal, E.; Salim, K.A.; Lim, L.B. Phytochemical screening, total phenolics and antioxidant activities of bark and leaf extracts of Goniothalamus velutinus (airy shaw) from brunei darussalam. J. King Saud Univ.-Sci. 2015, 27, 224-232. [CrossRef]

71. Yong, Y.; Saleem, A.; Guerrero-Analco, J.A.; Haddad, P.S.; Cuerrier, A.; Arnason, J.T.; Harris, C.S.; Johns, T. Larix laricina bark, a traditional medicine used by the cree of eeyou istchee: Antioxidant constituents and in vitro permeability across Caco-2 Cell Monolayers. J. Ethnopharmacol. 2016, 194, 651-657. [CrossRef]

72. Um, M.; Shin, G.-J.; Lee, J.-W. Extraction of total phenolic compounds from yellow poplar hydrolysate and evaluation of their antioxidant activities. Ind. Crops Prod. 2017, 97, 574-581. [CrossRef] 
73. El-Beshbishy, H.A.; Singab, A.N.B.; Sinkkonen, J.; Pihlaja, K. Hypolipidemic and antioxidant effects of Morus alba L. (egyptian mulberry) root bark fractions supplementation in cholesterol-fed rats. Life Sci. 2006, 78, 2724-2733. [CrossRef] [PubMed]

74. Valentín, L.; Kluczek-Turpeinen, B.; Willför, S.; Hemming, J.; Hatakka, A.; Steffen, K.; Tuomela, M. Scots pine (Pinus sylvestris) bark composition and degradation by fungi: Potential substrate for bioremediation. Bioresour. Technol. 2010, 101, 2203-2209. [CrossRef] [PubMed]

75. Ucar, M.B.; Ucar, G.; Pizzi, A.; Gonultas, O. Characterization of Pinus brutia bark tannin by MALDI-TOF MS and 13C NMR. Ind. Crops Prod. 2013, 49, 697-704. [CrossRef]

76. Meullemiestre, A.; Petitcolas, E.; Maache-Rezzoug, Z.; Chemat, F.; Rezzoug, S.A. Impact of ultrasound on solid-liquid extraction of phenolic compounds from maritime pine sawdust waste. kinetics, optimization and large scale experiments. Ultrason. Sonochem. 2016, 28, 230-239. [CrossRef]

77. Chupin, L.; Motillon, C.; Charrier-El Bouhtoury, F.; Pizzi, A.; Charrier, B. Characterisation of maritime pine (Pinus pinaster) bark tannins extracted under different conditions by spectroscopic methods, FTIR and HPLC. Ind. Crops Prod. 2013, 49, 897-903. [CrossRef]

78. Ku, C.; Sathishkumar, M.; Mun, S. Binding affinity of proanthocyanidin from waste Pinus radiata bark onto proline-rich bovine achilles tendon collagen type I. Chemosphere 2007, 67, 1618-1627. [CrossRef] [PubMed]

79. Frevel, M.A.; Pipingas, A.; Grigsby, W.J.; Frampton, C.M.; Gilchrist, N.L. Production, composition and toxicology studies of enzogenol Pinus radiata bark extract. Food Chem. Toxicol. 2012, 50, 4316-4324. [CrossRef] [PubMed]

80. Tantray, M.A.; Akbar, S.; Khan, R.; Tariq, K.A.; Shawl, A.S. Humarain: A new dimeric gallic acid glycoside from Punica granatum L. Bark. Fitoterapia 2009, 80, 223-225. [CrossRef]

81. Usenik, V.; Štampar, F.; Veberič, R. Anthocyanins and fruit colour in plums (Prunus domestica L.) during ripening. Food Chem. 2009, 114, 529-534. [CrossRef]

82. Bouras, M.; Chadni, M.; Barba, F.J.; Grimi, N.; Bals, O.; Vorobiev, E. Optimization of microwave-assisted extraction of polyphenols from Quercus bark. Ind. Crops Prod. 2015, 77, 590-601. [CrossRef]

83. Kim, K.H.; Moon, E.; Choi, S.U.; Kim, S.Y.; Lee, K.R. Polyphenols from the bark of Rhus verniciflua and their biological evaluation on antitumor and anti-inflammatory activities. Phytochemistry 2013, 92, 113-121. [CrossRef]

84. Zaiter, A.; Becker, L.; Petit, J.; Zimmer, D.; Karam, M.-C.; Baudelaire, É.; Scher, J.; Dicko, A. antioxidant and antiacetylcholinesterase activities of different granulometric classes of Salix alba (L.) bark powders. Powder Technol. 2016, 301, 649-656. [CrossRef]

85. Tewari, R.; Gupta, M.; Ahmad, F.; Rout, P.K.; Misra, L.; Patwardhan, A.; Vasudeva, R. Extraction, quantification and antioxidant activities of flavonoids, polyphenols and pinitol from wild and cultivated Saraca asoca bark using RP-HPLC-PDA-RI Method. Ind. Crops Prod. 2017, 103, 73-80. [CrossRef]

86. Jiménez-Sánchez, C.; Lozano-Sánchez, J.; Gabaldón-Hernández, J.A.; Segura-Carretero, A.; Fernández-Gutiérrez, A. RP-HPLC-ESI-QTOF/MS2 based strategy for the comprehensive metabolite profiling of Sclerocarya birrea (Marula) bark. Ind. Crops Prod. 2015, 71, 214-234. [CrossRef]

87. Subramanian, R.; Raj, V.; Manigandan, K.; Elangovan, N. Antioxidant activity of hopeaphenol isolated from Shorea roxburghii stem bark extract. J. Taibah Univ. Sci. 2015, 9, 237-244. [CrossRef]

88. Shastry Viswanatha, G.L.; Vaidya, S.K.; Ramesh, C.; Krishnadas, N.; Rangappa, S. Antioxidant and antimutagenic activities of bark extract of Terminalia arjuna. Asian Pac. J. Trop. Med. 2010, 3, 965-970. [CrossRef]

89. Yallappa, S.; Manjanna, J.; Sindhe, M.A.; Satyanarayan, N.D.; Pramod, S.N.; Nagaraja, K. Microwave assisted rapid synthesis and biological evaluation of stable copper nanoparticles using T. arjuna bark extract. Spectrochim. Acta A Mol. Biomol. Spectrosc. 2013, 110, 108-115. [CrossRef]

90. Kumar, V.; Sharma, N.; Sourirajan, A.; Khosla, P.K.; Dev, K. Comparative evaluation of antimicrobial and antioxidant potential of ethanolic extract and its fractions of bark and leaves of Terminalia arjuna from North-Western Himalayas, India. J. Tradit. Complement. Med. 2018, 8, 100-106. [CrossRef]

91. Bernardo, J.; Ferreres, F.; Gil-Izquierdo, Á.; Videira, R.A.; Valentão, P.; Veiga, F.; Andrade, P.B. In vitro multimodal-effect of Trichilia catigua A. Juss.(Meliaceae) bark aqueous extract in CNS targets. J. Ethnopharmacol. 2018, 211, 247-255. [CrossRef] [PubMed] 
92. Bolanle, J.D.; Adetoro, K.O.; Balarabe, S.A.; Adeyemi, O.O. Hepatocurative potential of Vitex doniana root bark, stem bark and leaves extracts against CCl4-induced liver damage in rats. Asian Pac. J. Trop. Biomed. 2014, 4, 480-485. [CrossRef] [PubMed]

93. Diouf, P.N.; Stevanovic, T.; Boutin, Y. The effect of extraction process on polyphenol content, triterpene composition and bioactivity of yellow birch (Betula alleghaniensis Britton) extracts. Ind. Crops Prod. 2009, 30, 297-303. [CrossRef]

94. Deng, Y.; Zhao, Y.; Padilla-Zakour, O.; Yang, G. Polyphenols, antioxidant and antimicrobial activities of leaf and bark extracts of Solidago canadensis L. Ind. Crops Prod. 2015, 74, 803-809. [CrossRef]

95. Kim, D.-H.; Kim, M.-J.; Kim, D.-W.; Kim, G.-Y.; Kim, J.-K.; Gebru, Y.A.; Choi, H.-S.; Kim, Y.-H.; Kim, M.-K. Changes of phytochemical components (urushiols, polyphenols, gallotannins) and antioxidant capacity during Fomitella fraxinea - mediated fermentation of Toxicodendron vernicifluum bark. Molecules 2019, 24, 683. [CrossRef] [PubMed]

96. Manach, C.; Williamson, G.; Morand, C.; Scalbert, A.; Rémésy, C. Bioavailability and bioefficacy of polyphenols in humans. A review of 97 bioavailability studies. Am. J. Clin. Nutr. 2005, 81, $230-242$. [CrossRef]

97. Giftson, J.S.; Jayanthi, S.; Nalini, N. Chemopreventive efficacy of gallic acid, an antioxidant and anticarcinogenic polyphenol, against 1, 2-dimethyl hydrazine induced rat colon carcinogenesis. Investig. New Drugs 2010, 28, 251-259. [CrossRef] [PubMed]

98. Velmurugan, B.; Rathinasamy, B.; Lohanathan, B.; Thiyagarajan, V.; Weng, C.-F. Neuroprotective role of phytochemicals. Molecules 2018, 23, 2485. [CrossRef]

99. Lee, S.-H.; Kim, Y.-J.; Kwon, S.-H.; Lee, Y.-H.; Choi, S.-Y.; Park, J.-S.; Kwon, H.-J. Inhibitory effects of flavonoids on TNF- $\alpha$-induced IL-8 gene expression in HEK 293 cells. BMB Rep. 2009, 42, 265-270. [CrossRef] [PubMed]

100. Tanase, C.; Cosarca, S.; Toma, F.; Mare, A.; Cosarca, A.; Man, A.; Miklos, A.; Imre, S. Antibacterial activities of spruce bark (Picea abies L.) extract and its components against human pathogens. Rev. Chim. 2018, 69, $1462-1467$.

101. Tănase, C.; Coşarcă, S.; Toma, F.; Mare, A.; Man, A.; Miklos, A.; Imre, S.; Boz, I. Antibacterial activities of beech bark (Fagus sylvatica L.) polyphenolic extract. Environ. Eng. Manag. J. EEMJ 2018, 17, 877-884. [CrossRef]

102. Choi, S.J.; Lee, M.Y.; Jo, H.; Lim, S.S.; Jung, S.H. Preparative isolation and purification of neuroprotective compounds from Rhus verniciflua by high speed counter-current chromatography. Biol. Pharm. Bull. 2012, 35, 559-567. [CrossRef] [PubMed]

103. Veerapur, V.; Thippeswamy, B.; Prabhakar, K.; Nagakannan, P.; Shivasharan, B.; Bansal, P.; Sneha, S.; Mishra, B.; Priyadarsini, K.; Unnikrishnan, M. Antioxidant and renoprotective activities of Ficus racemosa Linn. stem bark: Bioactivity guided fractionation study. Biomed. Prev. Nutr. 2011, 1, 273-281. [CrossRef]

104. Kosalec, I.; Kremer, D.; Locatelli, M.; Epifano, F.; Genovese, S.; Carlucci, G.; Randić, M.; Končić, M.Z. Anthraquinone profile, antioxidant and antimicrobial activity of bark extracts of Rhamnus alaternus, $R$. fallax, R. intermedia and R. pumila. Food Chem. 2013, 136, 335-341. [CrossRef] [PubMed]

105. Jitta, S.R.; Daram, P.; Gourishetti, K.; Misra, C.; Polu, P.R.; Shah, A.; Shreedhara, C.; Nampoothiri, M.; Lobo, R. Terminalia tomentosa bark ameliorates inflammation and arthritis in carrageenan induced inflammatory model and freund's adjuvant-induced arthritis model in rats. J. Toxicol. 2019, 2019, 1-11. [CrossRef] [PubMed]

(C) 2019 by the authors. Licensee MDPI, Basel, Switzerland. This article is an open access article distributed under the terms and conditions of the Creative Commons Attribution (CC BY) license (http://creativecommons.org/licenses/by/4.0/). 